\title{
EDITORIAL
}

\section{Breast Ductal Carcinoma in Situ}

\section{Precursor to Invasive Breast Cancer}

\author{
William B. Coleman
}

From the American Society for Investigative Pathology, Rockville, Maryland

This special Breast Ductal Carcinoma in Situ Theme Issue of The American Journal of Pathology includes four reviews covering major topics related to breast ductal carcinoma in situ (DCIS), which is a major health problem for women in the United States and worldwide. A focus on breast DCIS is warranted given its common occurrence, generally accepted precursor-product relationship with invasive breast cancer, and the need to treat DCIS patients appropriately to minimize the risk of progression to invasive disease. With an expanded understanding of the basic mechanisms that contribute to the development of DCIS and its progression to invasive breast cancer, key molecular pathways and effector molecules can be identified and exploited as biomarkers and in personalized approaches for treatment of the DCIS patient.

\section{Ductal Carcinoma in Situ-A Premalignant Breast Lesion}

DCIS of the breast is a pathologic lesion composed of abnormal epithelial cells (presumptive malignant cells) that are entirely confined within the breast ducts, surrounded by an intact myoepithelial cell layer and basement membrane structure (Figure 1A). ${ }^{1-3}$ The neoplastic cells that are observed in DCIS lesions closely resemble the microscopic appearance of the neoplastic cells composing invasive breast cancers (Figure 1, B and C). DCIS is thought to develop over a long period of time (years), beginning in the terminal duct lobular unit through distinct stages that reflect increasing abnormal cellular growths (Figure 1A). ${ }^{4}$ The neoplastic cells composing the DCIS lesion display characteristics of malignant cells, but they are contained within the normal ductular structure, lacking invasion of the myoepithelial cell layer and basement membrane (Figure 1A). The distinction between
DCIS and invasive breast cancer reflects the absence or presence (respectively) of invasion through the myoepithelial cell layer and basement membrane. With the emergence of an invasive element, the pathologic classification of the lesion becomes DCIS with invasive ductular carcinoma. Hence, in this model, DCIS represents a direct precursor to invasive breast cancer. ${ }^{4}$ The suggestion that DCIS is a precursor to invasive breast cancer is supported by histopathological observations over the past four decades, ${ }^{4}$ as well as more recent studies that have examined molecular similarities between DCIS lesions and breast cancers in the same patient. ${ }^{2,5,6}$ DCIS is considered to be non-life threatening because of the lack of an invasive component that can spread to adjacent tissue sites and/or metastasize to distant organs. Nevertheless, DCIS is an important pathologic lesion to detect and diagnose because it represents the immediate precursor to potentially lethal invasive breast cancers. ${ }^{4}$

In this issue, Sanati ${ }^{7}$ describes the histomorphologic features of breast DCIS lesions and various biomarkers that can be used to characterize DCIS lesions and predict their aggressiveness. Breast DCIS is pathologically classified on the basis of architectural patterns or histologic type. The major division of breast DCIS subtypes is comedo (with central necrosis) and noncomedo (Figure 1). ${ }^{8}$ Histologic types among noncomedo breast DCIS include papillary, solid, micropapillary, and cribriform. ${ }^{8}$ However, most DCIS cases are classified as DCIS not otherwise specified, which

\footnotetext{
Accepted for publication March 12, 2019.

Disclosures: None declared.

W.B.C. is the Special Editor for the Breast Ductal Carcinoma in Situ Theme Issue.

Address correspondence to William B. Coleman, Ph.D., American Society for Investigative Pathology, 1801 Rockville Pike, Ste. 350, Rockville, MD 20852. E-mail: wbcoleman@asip.org.
} 


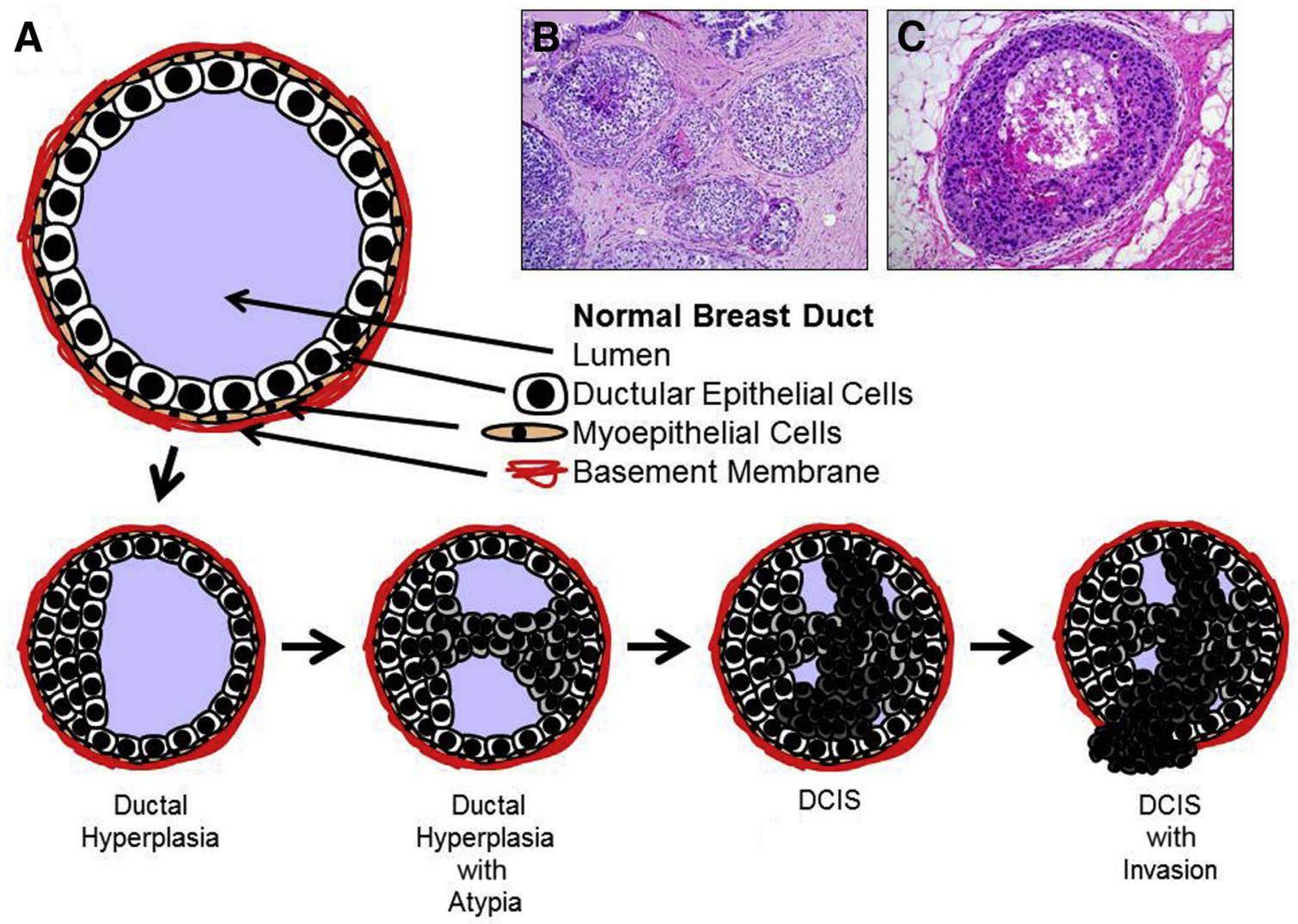

Figure 1 A: Natural history of breast ductal carcinoma in situ (DCIS) and progression to invasive breast cancer. The development of DCIS begins in the normal breast duct, characterized by a lumen surrounded by a single layer of differentiated breast ductular epithelial cells, which is supported by myoepithelial cells and basement membrane extracellular matrix. In response to some insulting event, altered cells begin to proliferate to form a hyperplastic lesion. With accumulation of additional alterations in the incipient neoplastic cells, atypical ductal hyperplasia develops. Over time, frank neoplastic cells proliferate to encompass the entire duct. This lesion is referred to as ductal carcinoma in situ because the presumptive malignant cells remain contained within the duct with an intact myoepithelial cell layer and basement membrane structures. On invasion through the myoepithelial cell layer and basement membrane, the lesion is classified as invasive ductal carcinoma. This schematic is adapted from the model of Wellings and Jensen. ${ }^{4} \mathbf{B}$ : Histology of noncomedo DCIS, demonstrating a solid growth pattern (hematoxylin and eosin stain). The images shown in A and B were provided by Dr. Benjamin Calhoun (Department of Pathology and Laboratory Medicine, University of North Carolina School of Medicine, Chapel Hill, NC). C: Histology of comedo DCIS, showing central necrosis (hematoxylin and eosin stain). Original magnification: $\times 10(\mathbf{B}) ; \times 20(\mathbf{C})$.

complicates studies focused on disease progression and long-term patient outcomes associated with specific DCIS subtypes. Nevertheless, comedo DCIS is generally considered to be a higher-grade lesion with a high nuclear grade and high rates of cellular proliferation compared with noncomedo DCIS subtypes. ${ }^{8}$ Sanati ${ }^{7}$ also discusses various genetic features of breast DCIS lesions and identification of intrinsic subtypes on the basis of gene expression patterns. Variations in molecular characteristics significantly contribute to the heterogeneity of DCIS lesions observed clinically and are likely to account for the varied clinical behaviors observed from patient to patient. Similar to invasive breast cancer, most (approximately $72 \%$ ) of DCIS lesions are estrogen receptor positive $\left(\mathrm{ER}^{+}\right){ }^{8}$ DCIS lesions have been characterized through immunohistochemical staining for ER, progesterone receptor, human epidermal growth factor receptor 2 (HER2), and related gene products, revealing that DCIS lesions can be subdivided into luminal A (ER $\left.{ }^{+} / \mathrm{HER} 2^{-}, 49 \%\right)$, luminal B/HER2 ${ }^{-}\left(\mathrm{ER}^{+} / \mathrm{Ki}-67\right.$ high, 9\%), luminal B/HER2 ${ }^{+}\left(\mathrm{ER}^{+} /\right.$
$\left.\mathrm{HER}^{+}, 17 \%\right)$, HER2 enriched $\left(\mathrm{ER}^{-} / \mathrm{HER}^{+}, 16 \%\right)$, and triple negative (ER ${ }^{-} /$progesterone receptor negative/ HER2 $\left.{ }^{-}, 7 \%\right){ }^{9}$ Likewise, gene expression studies have revealed the intrinsic subtypes among DCIS specimens, although a substantial proportion $(20 \%)$ do not conform to any of these subtypes, indicating a mixed/heterogeneous composition. ${ }^{10}$ Also in this issue, Hannafon and Ding ${ }^{11}$ describe the current understanding of the role of miRNAs in the genesis and natural history of breast DCIS. Changes in miRNA expression accompany the progression of DCIS to invasive breast cancer through modification of oncogenic and tumor-suppressive patterns of gene expression. ${ }^{11}$ miRNAs also represent candidate biomarkers for prediction of aggressiveness of DCIS lesions and/or as therapeutic targets to prevent progression of DCIS to invasive cancer.

\section{Breast DCIS Is a Significant Health Problem}

Invasive breast cancer (invasive ductal carcinoma) is a major health issue for women in the United States and 
worldwide. In the United States, an estimated 268,600 new cases of invasive breast cancer will be diagnosed in $2019 .{ }^{12}$ Hence, breast cancer is by far the most frequently occurring invasive cancer among women (not including nonmelanoma skin cancer). Breast DCIS is also a commonly occurring neoplastic lesion, with an estimated 62,930 new cases diagnosed in 2019. ${ }^{12}$ This reflects more new cases of breast DCIS than all other invasive forms of cancer in women, with the exception of cancers of the breast, lung, and colorectum. ${ }^{12}$ Breast DCIS is a premalignant lesion (lacking invasive elements), but this form of breast disease comprises nearly $20 \%$ of all neoplastic breast lesions diagnosed. ${ }^{12}$ The development of breast DCIS is age dependent, similar to invasive breast cancer. Most cases of DCIS are diagnosed in postmenopausal women ( $>50$ years of age), and the highest incidence occurs in women between the ages of 65 and 75 years (NIH Surveillance, Epidemiology, and End Results Program, https://seer.cancer.gov/csr/1975_2015, last accessed February 15, 2019). DCIS incidence varies with race/ethnicity, similar to invasive breast cancer. The incidence of DCIS diagnosis is similar among white women, African American women, and Asian Pacific Islander women, and it is less frequent among Hispanic women and Native American women (https://seer.cancer. gov/csr/1975_2015).

Before the mid-1980s, DCIS was rarely detected and diagnosed (approximately 10 cases per 100,000 in women $>50$ years old; https://seer.cancer.gov/csr/1975_2015). With the advent of population screening using mammography, the frequency of DCIS diagnosis increased sharply. In 2015, the incidence of DCIS was $>85$ cases per 100,000 in women $>50$ years old (https://seer.cancer.gov/csr/1975_2015). Although DCIS can present clinically in the patient as a palpable mass or as nipple discharge, it is now frequently detected by the appearance of calcifications during mammographic screening. ${ }^{8}$ Histologic confirmation of DCIS is achieved through needle biopsy, followed by surgical excision and definitive examination of lesion pathology. Invasive breast ductal carcinoma is found after surgery in $25 \%$ of DCIS cases diagnosed on the basis of core biopsy. ${ }^{8}$ The finding of invasive ductal carcinoma on pathologic evaluation of surgically resected DCIS alters the patient's diagnosis to stage 1 breast cancer. Lymph node dissection is not indicated in patients with pure DCIS. However, identification of an invasive component would lead to lymph node evaluation for purposes of staging. DCIS lesions that are larger, with an intermediate or high nuclear grade and negative hormone receptor status, are more likely to harbor an invasive element indicative of disease progression. ${ }^{13,14}$

The expanded use of screening mammography has resulted in dramatic increases in the frequency of detection and diagnosis of breast DCIS in recent decades. Although DCIS is considered to be a precursor to invasive breast cancer, data are lacking as to the proportion of breast DCIS lesions that will progress to invasive cancer in the absence of treatment. However, some insights into the frequency of progression from DCIS to invasive breast cancer have emerged from long-term studies of patients who were not treated because of misclassification of biopsy specimens as benign. These studies show that $20 \%$ to $>50 \%$ of untreated patients were diagnosed with invasive breast cancer over a period of $\geq 10$ years. ${ }^{1,15-18}$ Additional insight into the natural history of DCIS and progression to invasive breast cancer emerged from studies of the so-called Nashville cohort. ${ }^{19}$ Among this group of patients, 16 of $45(36 \%)$ developed invasive breast cancer in the same breast and quadrant where the incident DCIS lesion was identified. ${ }^{19}$ Among the DCIS patients who developed invasive breast cancer, 11 of 16 (69\%) experienced disease progression within 10 years of their DCIS biopsy, and the others were diagnosed with invasive breast cancer at 12, 23, 25, 29, and 42 years after their DCIS biopsy. ${ }^{19}$ Furthermore, 7 of 16 patients (44\%) diagnosed with invasive breast cancer developed metastatic disease, resulting in death over a period of 1 to 7 years after diagnosis. ${ }^{19}$ Some studies have followed the long-term outcomes of patients with small DCIS lesions, treated with surgical excision only, and wide margins. In these studies, the aggressiveness of the incipient DCIS lesion was associated with tendency for disease progression: $10.5 \%$ of patients in the low/intermediate-grade group developed invasive breast cancer within 7 years, versus $18 \%$ of patients in the high-grade group. ${ }^{20,21}$ In a subset of patients, the 10-year rates for development of invasive breast cancer were $3.7 \%$ for the low-grade group, $12.3 \%$ for the intermediate-grade group, and $19.2 \%$ for the high-grade group. ${ }^{20,21}$ All of these studies highlight the fact that not all breast DCIS lesions will progress to invasive breast cancer and the importance of identifying which patients are likely to experience disease progression and which ones will not. This important distinction ensures that patients who are likely to progress will receive appropriate clinical intervention and those who are unlikely to progress are not overtreated. In this issue, Nofech-Mozes et $\mathrm{al}^{22}$ describe the use of Oncotype DX DCIS (Genomic Health, Redwood City, CA) in the assessment of DCIS lesions in patients who undergo breast-conserving surgery to predict risk of local recurrence of DCIS or progression to invasive cancer. Oncotype DX DCIS is a commercially available multigene assay that includes seven cancer-related genes and five reference genes, whose results can assist in clinical decision making in subsets of DCIS patients with low risk of progression. ${ }^{22}$ Oncotype DX DCIS identifies patients with low risk of disease progression who can reasonably avoid adjuvant chemotherapy after surgery, reducing the possibility of overtreatment.

\section{The Clinical Challenge of Breast DCIS}

The clinical challenge associated with DCIS patients reflects the question of which patients require treatment and how aggressive this treatment should be. ${ }^{23}$ It is clear that breast 
DCIS development is associated with similar risk factors as those associated with invasive breast cancer, breast DCIS frequently accompanies invasive breast cancer, and some of the biology of these lesions mirrors that of invasive breast cancer. Furthermore, significant numbers of patients who are treated for breast DCIS will develop invasive breast cancer as a product of disease recurrence and progression. However, it is also apparent that not all patients with a diagnosis of breast DCIS will progress to invasive breast cancer. Hence, the major clinical challenge associated with breast DCIS is to identify low-risk patients who can be treated with surgery only, while sparing these patients from radiation therapy. ${ }^{24}$ Despite attempts to develop molecular tests to identify DCIS lesions that are likely to progress to invasive breast cancer, ${ }^{25}$ such molecular assays currently lack clinical utility. Development of sensitive and specific molecular diagnostics that can be used in conjunction with more traditional pathologic evaluation to identify low-risk patients will require a more complete understanding of the pathobiology of this neoplastic breast lesion. In this issue, Shee et $\mathrm{al}^{26}$ discuss existing molecular biomarkers for precision medicine approaches to DCIS management and approaches to identification of new biomarkers for DCIS. With effective biomarkers and biomarker-based molecular assays, overtreatment of breast DCIS might be avoided by identifying the patients who are most likely to progress to invasive cancer (or, conversely, are unlikely to undergo disease progression). ${ }^{26}$

\section{References}

1. Allred DC: Ductal carcinoma in situ: terminology, classification, and natural history. J Natl Cancer Inst Monogr 2010, 41:134-138

2. Cowell CF, Weigelt B, Sakr RA, Ng CKY, Hicks J, King TA, ReisFilho JS: Progression from ductal carcinoma in situ to invasive breast cancer: revisited. Mol Oncol 2013, 7:859-869

3. Lopez-Garcia MA, Geyer FC, Lacroix-Triki M, Marchio C, ReisFilho JS: Breast cancer precursors revisited: molecular features and progression pathways. Histopathology 2010, 57:171-192

4. Wellings RR, Jensen HM: On the origin and progression of ductal carcinoma in the human breast. J Natl Cancer Inst 1973, 50:1111-1118

5. Bukhardt L, Grob TJ, Hermann I, Burandt E, Choschzick M, Janicke F, Muller V, Bokmeyer C, Simon R, Sauter G, Wilczak W, Lebeau A: Gene amplification in ductal carcinoma in situ of the breast. Breast Cancer Res Treat 2010, 123:757-765

6. Schultz S, Bartsch H, Sotlar K, Petat-Dutter K, Bonin M, Kahlert S, Harbeck N, Vogel U, Seeger H, Fehm T, Neubauer HJ: Progressionspecific genes identified in microdissected formalin-fixed and paraffinembedded tissue containing matched ductal carcinoma in situ and invasive ductal breast cancers. BMC Med Genomics 2018, 11:80

7. Sanati S: Morphologic and molecular features of breast ductal carcinoma in situ. Am J Pathol 2019, 189:946-955

8. Ward EM, DeSantis CE, Lin CC, Kramer JL, Jemal A, Kohler B, Brawley OW, Gansler T: Cancer statistics: breast cancer in situ. CA Cancer J Clin 2015, 65:481-495

9. Zhou W, Jirstrom K, Amini RM, Fjallskog ML, Sollie T, Lindman H, Sorlie T, Blomqvist C, Warnberg F: Molecular subtypes in ductal carcinoma in situ of the breast and their relation to prognosis: a population-based cohort study. BMC Cancer 2013, 13:512

10. Allred CD, Wu Y, Mao S, Nagtegaal ID, Lee S, Perou CM, Mohsin SK, O'Connell P, Tsimelzon A, Medina D: Ductal carcinoma in situ and the emergence of diversity during breast cancer evolution. Clin Cancer Res 2008, 14:370-378

11. Hannafon BN, Ding W-Q: Functional role of microRNAs in the progression of breast ductal carcinoma in situ. Am J Pathol 2019, 189: 966-974

12. Seigel RL, Miller KD, Jemal A: Cancer statistics, 2019. CA Cancer J Clin 2019, 69:7-34

13. Kantor O, Winchester DJ: Breast conserving therapy for DCIS: does size matter? J Surg Oncol 2014, 110:75-81

14. Lee SK, Yang JH, Woo SY, Lee JE, Nam SJ: Nomogram for predicting invasion in patients with a preoperative diagnosis of ductal carcinoma in situ of the breast. Br J Surg 2013, 100: $1756-1763$

15. Eusebi V, Feudale E, Foschini MP, Micheli A, Conti A, Riva C, Di Palma S, Rilke F: Long-term follow-up of in situ carcinoma of the breast. Semin Diagn Pathol 1994, 11:223-235

16. Sanders ME, Schuyler PA, Dupont WD, Page DL: The natural history of low-grade ductal carcinoma in situ of the breast in women treated by biopsy only revealed over 30 years of long-term follow-up. Cancer 2005, 103:2481-2484

17. Collins LC, Tamimi RM, Baer HJ, Connolly JL, Colditz GA, Schnitt SJ: Outcome of patients with ductal carcinoma in situ untreated after diagnostic biopsy: results from the Nurses' Health Study. Cancer 2005, 103:1778-1784

18. Erbas B, Provenzano E, Ames J, Gertig D: The natural history of ductal carcinoma in situ of the breast: a review. Breast Cancer Res Treat 2006, 97:135-144

19. Sanders ME, Schuyler PA, Simpson JF, Page DL, Dupont WD: Continued observation of the natural history of low-grade ductal carcinoma in situ reaffirms proclivity for local recurrence even after more than 30 years of follow-up. Mod Pathol 2015, 28:662-669

20. Hughes LL, Wang M, Page DL, Gray R, Solin LJ, Davidson NE, Lowen MA, Ingle JN, Recht A, Wood WC: Local excision alone without irradiation for ductular carcinoma in situ of the breast: a trial of the Eastern Cooperative Oncology Group. J Clin Oncol 2009, 27: $5319-5324$

21. Morrow M, Schnitt SJ, Norton L: Current management of lesions associated with an increased risk of breast cancer. Nat Rev Clin Oncol 2015, 12:227-238

22. Nofech-Mozes S, Hanna W, Rakovitch E: Molecular evaluation of breast ductal carcinoma in situ with Oncotype DX DCIS. Am J Pathol 2019, 189:975-980

23. Sagara Y, Julia W, Golshan M, Masakazu T: Paradigm shift toward reducing overtreatment of ductal carcinoma in situ of breast. Front Oncol 2017, 7:192

24. Shah C, Vicini FA, Berry S, Julian TB, Wilkinson JB, Shaitelman SF, Khan A, Finkelstein SE, Goldstein N: Ductal carcinoma in situ of the breast: evaluating the role of radiation therapy in the management and attempts to identify low-risk patients. Am J Clin Oncol 2015, 38: $526-533$

25. Solin LJ, Gray R, Baehner FL, Butler SM, Hughes LL, Yoshizawa C, Cherbacvaz DB, Shak S, Page DL, Sledge GW Jr, Davidson NE, Ingle JN, Perez EA, Wood WC, Sparano JA, Badve S: A multigene expression assay to predict local recurrence risk for ductal carcinoma in situ of the breast. J Natl Cancer Inst 2013, 105:701-710

26. Shee K, Muller KE, Marotti J, Miller TW, Wells WA, Tsongalis GJ: Ductal carcinoma in situ biomarkers in a precision medicine era: current and future molecular-based testing. Am J Pathol 2019, 189: 956-965 